\title{
Design of a CMOS Temperature Sensor with Current Output
}

\author{
ARJAN KÖLLING*, FRANS BAK, PIET BERGVELD and EVERT SEEVINCK \\ Faculty of Electrical Engineering, Unwersity of Twente, $P O$ Box 217, 7500 AE Enschede (The Netherlands)
}

\begin{abstract}
In this paper a CMOS temperature-to-current converter is presented of which the output current is the difference between a PTC current and an NTC current The PTC current is derived from a PTAT cell, while the NTC current is derived from a threshold voltage reference source It is shown that this way of current subtraction diminishes to a certain extent the effects of the thermal dependence of the resistors used
\end{abstract}

\section{Introduction}

Although the operation of most electronic components and circuits is degraded by temperature fluctuations of the operating environment, they offer very good prospects for the realization of electronic temperature sensors For example, the thermal behaviour of the pn-junction is often used in electronic temperature sensors [1] For the realization of a temperature-to-current converter in CMOS technology, MOS transistors operating in weak inversion mode can be used, since they appear to behave similarly to pn-junctions, with respect to their thermal behaviour Thus, a PTAT cell can also be made in CMOS technology

\section{The PTAT Principle}

The combination of two equal diodes, forwardly biased by currents with a fixed ratio $N$, as shown in Fig 1(a), produces a voltage difference between the diodes that is proportional to the absolute temperature (PTAT) It can be calculated that the voltage $V_{\mathrm{PTAT} \text { a }}$ for $I_{\mathrm{D} 2}=N I_{\mathrm{D} 1}$ equals

$V_{\text {PTAT a }}=\frac{k T}{q} \ln N$

which is proportional to the absolute temperature

*Present address Texas Instruments Holland B V , P O Box 43, 7600 AA Almelo, The Netherlands
In the circuit shown in Fig 1(b), the voltage $V_{\text {PTAT b }}$ across the resistor $R$ is also proportional to the absolute temperature, if both MOSTs $\operatorname{Tr}_{1}$ and $\mathrm{Tr}_{\mathbf{2}}$ are operating in weak inversion An MOS transistor operates in weak inversion if the drain current $I_{\mathrm{D}}$ is much smaller than the weak inversion limit

$I_{\mathrm{D}} \ll \beta U_{\mathrm{T}}^{2}$

with

$\beta=\frac{W}{L} \frac{\mu C_{\mathrm{OX}}}{2}$ and $U_{\mathrm{I}}=\frac{k T}{q}$

where $W=$ gate width, $L=$ gate length, $\mu=$ inversion layer mobility, $C_{\text {ox }}=$ gate oxide capacitance/ $\square$ Under weak inversion conditions the drain current for operation in saturated mode can be described as [2]

$I_{\mathrm{D}}=K \beta U_{\mathrm{T}}^{2} \exp \left[\left(V_{\mathrm{G}}-V_{\mathrm{T}}-n V_{\mathrm{S}}\right) / n U_{\mathrm{T}}\right]$

where $V_{\mathrm{G}}=$ gate voltage, $V_{\mathrm{T}}=$ threshold voltage, $V_{\mathrm{s}}=$ source voltage, $K$ and $n=$ constants Now the voltage $V_{\text {PTAT, b }}$, appeaning in the crcuit shown in Fig 1(b), can be calculated using eqn (3) If both transistors share a common substrate, then $V_{\text {PTAT o }}$ equals

$$
V_{\text {PTAT b }}=\frac{k T}{q} \ln \left[\frac{(W / L)_{2}}{N(W / L)_{1}}\right]
$$

which again is proportional to the absolute temperature

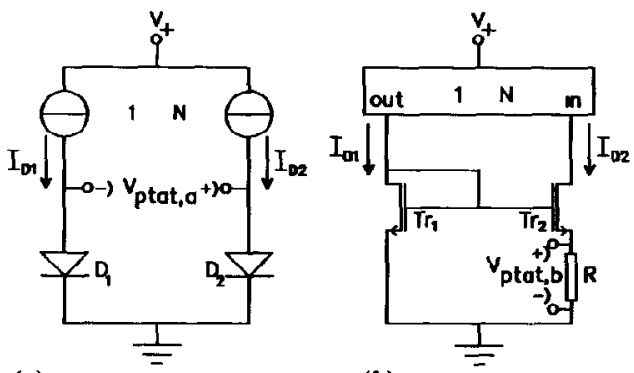

(a)

(b)

Fig 1 (a) Basic PTAT-voltage source (b) Weak inversion MOST PTAT-voltage source

(C) Elsevier Sequoia/Printed in The Netherlands 
Although the voltage across the resistor $R$ is proportional to the absolute temperature, the current $I_{\mathrm{D} 2}$ through the resistor, which equals $V_{\text {PTAT b }} / R$, will show nonlinear behaviour, due to the thermal dependence of the resistor In order to describe the thermal behaviour of a resistor in a restricted temperature range, the resistance around a certain temperature $T_{\mathrm{z}}$ can be described by means of a Taylor series

$$
R(T)=R\left(T_{z}\right)\left(1+\alpha_{1}\left(T-T_{z}\right)+\alpha_{2}\left(T-T_{z}\right)^{2}+\Delta\right)
$$

with $\alpha_{i}$ equal to the weight factor of the $l^{\text {th }}$ order term and $\Delta$ representing the sum of the higherorder terms

In general, a temperature-dependent current, $I_{1}(T)$, derived from a temperature-dependent voltage, $V_{1}(T)$, by means of a temperature resistor, $R_{1}(T)$, can be expressed as

$$
I_{1}(T)=V_{1}(T) / R_{1}(T)
$$

Using expression (5), the thermal dependence of $I_{1}$ can be expressed as

$$
\begin{aligned}
\frac{\partial I_{1}(T)}{\partial T}= & I_{1}(T)\left[\frac{1}{V_{1}(T)} \frac{\partial V_{1}}{\partial T}-\frac{R_{1}\left(T_{z}\right)}{R_{1}(T)}\right. \\
& \left.\times\left[\alpha_{1}+2 \alpha_{2}\left(T-T_{z}\right)+\frac{\partial \Delta}{\partial T}\right]\right]
\end{aligned}
$$

At the temperature $T=T_{\mathrm{z}}$ expression (7) equals

$\left.\frac{\partial I_{1}}{\partial T}\right|_{T_{z}}=I_{1}\left(T_{z}\right)\left[\left.\frac{1}{V_{1}\left(T_{z}\right)} \frac{\partial V_{1}}{\partial T}\right|_{T_{z}}-\alpha_{1}\right]$

From expression (8) it follows that the first-order temperature dependence of the resistor $R_{1}$, represented by the coefficient $\alpha_{1}$, can influence the temperature coefficient of the current $I_{1}\left(T_{\mathrm{z}}\right)$ extensively For example, in the case of an integrated n-diffusion resistor (with a donor concentration $N_{\mathrm{d}}=2 \times 10^{16} / \mathrm{cm}^{3}$ ) we measured a value of $\alpha_{1}$ of about 0004 per $\mathrm{K}$ at room temperature In the case of a PTAT voltage source, $V_{1}(T)$ equals $A T$ ( $A$ is a constant) so expression (8) equals for this example

$\left.\frac{\partial I_{1}}{\partial T}\right|_{T_{z}}=I_{1}\left(T_{z}\right)\left[\frac{1}{T_{z}}-\alpha_{1}\right]$

At room temperature, $1 / T_{z}$ equals about 0003 , which is smaller than $\alpha_{1}$, so it is noted that the sign of the temperature coefficient of the output current of this circuit is negative

In general, it can be concluded that a diffusion resistor in a PTAT cell always results in a current which is nonlinear with respect to the temperature For this reason a more sophisticated temperature-to-current converter has been developed in which the influence of the thermal dependence of the resistor on the temperature coefficient of the output current is decreased The principle of this $T-I$-converter is described in the next section

\section{Temperature-to-current Converter Design with Improved Behaviour}

As shown in the previous paragraph, the infiuence of the resistor determines to a great extent the temperature behaviour of the temperature-tocurrent conversion in a CMOS PTAT cell A better temperature-to-current conversion can be obtained by creating a second temperaturedependent current

$$
I_{2}(T)=V_{2}(T) / R_{2}(T)
$$

and subtracting this current from the current $I_{1}$, as shown in Fig 2 The principle operation of a temperature sensor based on the difference between two temperature-dependent current sources has been described by Meijer [3] The quintessence of this principle is the possibility to create an output current on an arbitrary temperature scale

In this section it will be shown that application of this principle, using two nonlinear temperaturedependent current sources in which the nonlinearities are caused by identical resistors, will improve the performance drastically, with respect to the single PTAT cell

Since the current $I_{1}$ is derived from a PTAT cell, this current can be expressed as

$I_{1}(T)=\frac{A T}{R_{1}(T)}$

The constant $A$ represents the temperature coefficient of the PTAT voltage

The current source $I_{2}(T)$, derived from a voltage source with a negative temperature coefficient, can be described by

$I_{2}(T)=\frac{V_{\mathrm{r}}-\gamma T}{R_{2}(T)}$

where $V_{\mathrm{r}}$ represents the value of the voltage source at $T=0 \mathrm{~K}$ and $\gamma$ represents the temperature coefficient of this source

Both current $I_{1}$ and $I_{2}$ are graphically represented in Fig 3(a) In this graph the values of the resistors $R_{1}$ and $R_{2}$ are assumed to be constant and equal to their value at $T=T_{z}$, in order to

$$
I_{1}-I_{p t c}=\frac{v_{p t o t}}{R_{1}} \mid I_{2}-I_{n t c}=\frac{v_{\text {ntc }}}{R_{2}} \bigodot_{I_{\text {out }}}=I_{1}-I_{2}
$$

Fig 2 Principle of temperature-to-current converter with improved behaviour 


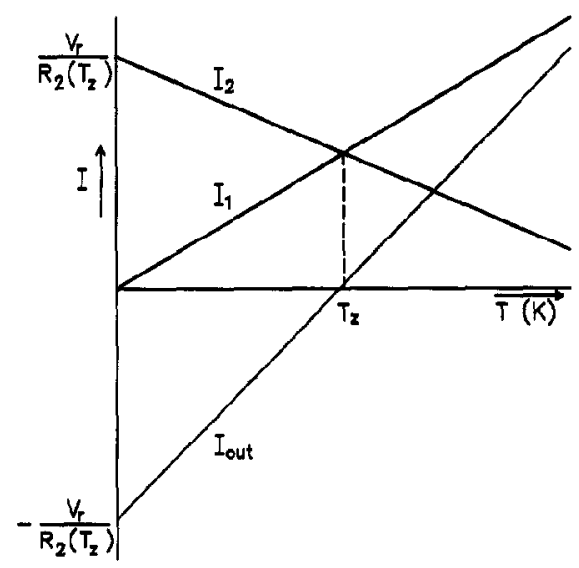

(a)

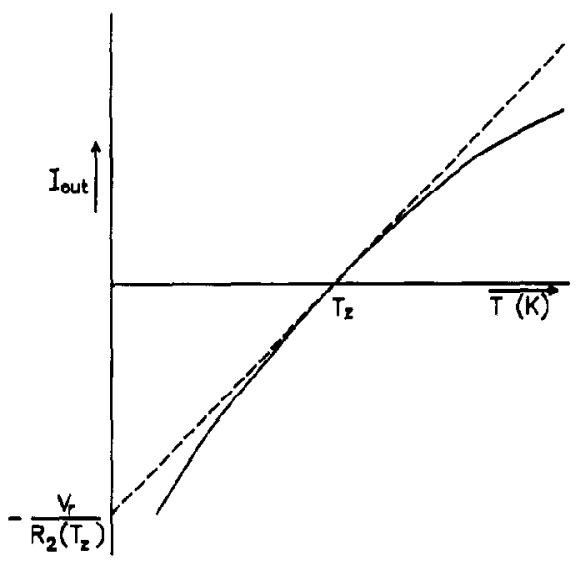

(b)

Fig 3 (a) Graphical representation of both thermaldependent currents and the difference current $I_{\text {out }}=I_{1}-I_{2}$ (b) Deviation of $I_{\text {out }}$ caused by thermal-dependent resistors

obtain a linearized expression for the difference current $I_{\text {out }}(T)=I_{1}(T)-I_{2}(T)$ at around $T=T_{z}$

It can easily be seen from $F_{1}$ 3(a) that a linearized expression for around $T=T_{\mathrm{z}}$ for the output current $I_{\text {out }}(T)$ equals

$I_{\text {out }}(T)=I_{1}(T)-I_{2}(T)=\frac{V_{r}}{T_{z} R_{2}\left(T_{z}\right)}\left(T-T_{z}\right)$

In order to estimate the influence of the thermal dependence of the resistors on the linearized expression (13), the constant value $R_{2}\left(T_{z}\right)$ is replaced by the Taylor estimation of $R_{2}$ around $T=T_{\mathrm{z}}$ Using this Taylor series estimation of the resistance of $R_{2}$ (expression (5)), expression (13) can be written as

$I_{\text {out }}\left(T-T_{z}\right)=\frac{V_{\mathrm{r}}}{T_{\mathrm{z}} R_{2}\left(T_{z}\right)\left(1+\alpha_{1}\left(T-T_{\mathrm{z}}\right)\right)}\left(T-T_{\mathrm{z}}\right)$ under the assumption that the high-order terms are dominated by the first order term $\alpha_{1}$

For small values of $\alpha_{1}\left(T-T_{z}\right)$, expression (14) can be estımated by

$I_{\text {out }}(T)=\frac{V_{\mathrm{r}}}{T_{\mathrm{z}} R_{2}\left(T_{\mathrm{z}}\right)}\left(T-T_{\mathrm{z}}\right)\left(1-\alpha_{1}\left(T-T_{\mathrm{z}}\right)\right)$

Equation (15) is graphically shown in Fig 3(b) From this Figure it can be seen that the most accurate results are obtanned if the reference temperature $T_{\mathrm{z}}$ is chosen in the middle of the desired measurement range

As already argued in the previous section, the current source $I_{1}$ derives its output current from the PTAT cell of which the voltage has a positive temperature coefficient (PTC source, eqn (4)) The current source $I_{2}$ derives its output current from a voltage with a negative temperature coefficient (NTC source), viz the threshold voltage reference, as described in the next section

\section{Threshold Voltage Reference}

It is well known that the threshold voltage $V_{\mathrm{T}}$ of an MOS transistor shows a negative temperature dependence [4] In the circuit shown in Fig 4, the negative temperature coefficient of the threshold voltage is applied to create a voltage over the resistor $R_{2}$ that also has a negative temperature coefficient, as will be shown below The MOST $M_{1}, F_{1 g} 4$, is biased to operate in weak inversion Thus, by application of expression (3) for the drain current of MOST $M_{1}$, the voltage $V_{\mathrm{G}}$ over resistor $R_{2}$ can be calculated

$V_{\mathrm{G}}=V_{\mathrm{T}}+\delta \quad$ with $\delta=n U_{\mathrm{T}} \ln \frac{I_{\mathrm{D}}}{K \beta U_{\mathrm{T}}^{2}}$

As mentioned before, the threshold voltage $V_{\mathrm{T}}$ shows a negative temperature coefficient and can

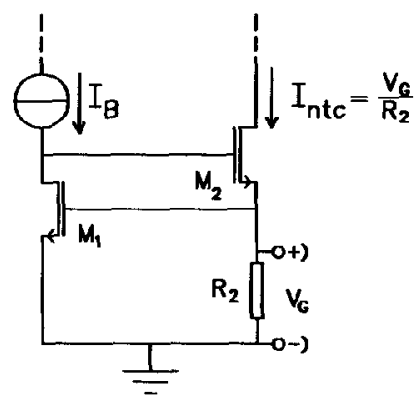

Fig 4 Threshold-voltage reference source 
be described in a small temperature range by

$V_{\mathrm{T}}(T)=V_{\mathrm{Te}}-\gamma T+\Delta(T)$

$\Delta(T)$ represents the high-order terms in this approximation, $V_{\text {Te }}$ can be interpreted as the extrapolated threshold voltage at $T=0 \mathrm{~K}$ Under these assumptions, the voltage $V_{\mathrm{G}}$ over $R_{2}$ can be described as

$V_{\mathrm{G}}=V_{\mathrm{Te}}-\gamma T+\epsilon$

with $\epsilon$ representing the deviation from the linearized behaviour

A disadvantage is clear the threshold voltage is not accurately reproducible and is sensitive to process variations Since a shift in the threshold voltage leads to a shift in the offset $V_{\mathrm{Te}}$ in expression (18), the reproducibility of the offset of the output voltage $V_{G}$ will be bad

\section{Realization of the Temperature-to-current Converter}

In this section the CMOS realization of the temperature-to-current converter, which operates according to the aforementioned principle, is described and its performance is discussed A simplified schematic of the realized temperatureto-current converter is shown in Fig 2 The resistors $R_{1}$ and $R_{2}$ are realized as integrated n-type-doped diffusion resistors In the first realization, the threshold voltage reference was used as the NTC source In the realized chip design, it was possible to perform measurements using integrated diffusion resistors as well as external resistors Temperature measurements, in the range from $298-333 \mathrm{~K}$, performed on this circuit are shown in Fig 5 Curve 1 of Fig 5 shows a measurement with external resistors, kept at a constant temperature In this case the output current of the circuit can be described by expression (13) The temperature coefficient was designed to be $84 \mathrm{nA} / \mathrm{K}$, and a value of $814 \mathrm{nA} / \mathrm{K}$ was measured The nonlinearity in the sensor signal was smaller than the measurement inaccuracy in the given temperature range

Curve 2 of Fig 5 shows measurement with integrated diffusion resistors In this case expression (15) describes the sensor signal The measured first-order temperature coefficient of the resistors equals $4250 \times 10^{-3} / \mathrm{K}$ With this very large temperature coefficient of the resistors, the nonlinear deviation of the output signal is evident in a temperature range from 308 to $318 \mathrm{~K}\left(35-45^{\circ} \mathrm{C}\right)$ a deviation of already $04 \mathrm{~K}$ is measured Although the nonlinearity is large, eqn (15) gives a very good estimation of this nonlinear behaviour In practice, this implies that accurate measurement

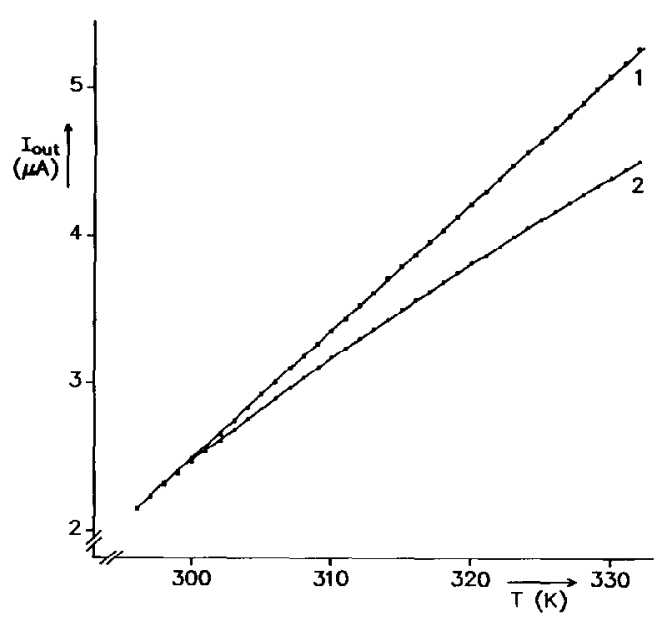

Fig 5 Temperature-current measurements 1 , external resistors kept at constant temperature, 2, diffusion resistors, integrated on the chip

results can be obtained after a two-point calibration is carried out, under the assumption that the first-order temperature coefficient of the resistors $\alpha_{1}$ is known The results from this calibration are used in expression (19) For example, for curve 2 of Fig 5 it is calculated in this way that

$I_{\text {out }}=845(T-2755)\left(1-4250 \times 10^{-6}(T-277)\right)$

(in nA with $T$ in $\mathrm{K}$ )

Expression (19) describes the behaviour of the sensor in this case with an accuracy of $005 \mathrm{~K}$ in the range from 298 to $333 \mathrm{~K}$

The reproducibility of this temperature sensor depends for a great part on the threshold-voltage variations, as mentioned earlier As stated then, a threshold-voltage shift results in a zero-point shift of the output current We measured thresholdvoltage variations over the same wafer of about $50 \mathrm{mV}$ This threshold-voltage shift results in a measured zero-point shift of about $15 \mathrm{~K}$ The expected threshold-voltage shift between samples from different batches is much larger Thus the zero point of this temperature sensor is hardly predictable

Another aspect with respect to the applicability of this temperature sensor is the reproducibility of the resistors In a second realization, we measured a deviation of $50 \%$ of the resistor values from the designed value Thus, the reproducibility of diffusion resistors, at least in the CMOS process used by us, seems questionable

\section{Conclusions}

In this paper it is shown that an MOS transistor operated in weak inversion offers good possi- 
bilities to be applied as a temperature-sensing element However, in order to obtain a temperature-dependent current, a single PTAT source and an integrated resistor do not give a satısfyıng result An improved temperature-to-current converter is presented that makes use of two integrated resistors and an extra temperature-dependent voltage source that features a negative temperature coefficient This principle offers the possibility of an output signal on an arbitrary temperature scale

However, a drawback appears to be that the threshold voltage reference source suffers from the low reproducibility of the threshold voltage of an MOST Another drawback of the presented temperature-to-current converter is the dependence on the absolute value of the integrated diffusion resistors Unfortunately, the sheet resistance of diffusion resistors is not very reproducible

An alternative for the integrated diffusion resistors can possibly be found in the application of thin-film resistors that should be deposited on the chip after the CMOS process is finished

\section{Acknowledgements}

The authors are grateful to A Kooy for processing the curcuits at the IC-processing facility of the University of Twente These investigations in the programme of the Foundation for Fundamental Research on Matter (FOM) have been supported by the Netherlands Technology Foundation (STW)

\section{References}

$1 \mathrm{G} \mathrm{C} \mathrm{M} \mathrm{Meijer,} \mathrm{Thermal} \mathrm{sensors} \mathrm{based} \mathrm{on} \mathrm{transistors,}$ Sensors and Actuators, 10 (1986) 103-126

2 E Vittoz and J Fellrath, CMOS analog crrcuits based on weak inversion operation, IEEE $J$ Solid-State Circuts, SC-12 (1977) 224-231

3 G C M Meıjer, A low-power easy-to-calibrate temperature transducer, IEEEJ Solid-State Circuts, SC-17(1982) 609613

$4 \mathrm{~L}$ Vadasz and A S Grove, Temperature dependence of MOS transistor charactenstics below saturation, IEEE Trans Electron Devices, ED-13 (1966) 863-866 